\title{
Development of a Contact-Potential-Type Phase Plate
}

\author{
H. Tamaki ${ }^{1}$, H. Kasai ${ }^{1}$, K. Harada ${ }^{1}$, Y. Takahashi ${ }^{1}$, and R. Nishi ${ }^{2}$. \\ 1. Central Research Laboratory, Hitachi, Ltd., 1-280, Higashi-Koigakubo, Kokubunji-shi, Tokyo 185-8601, Japan \\ 2. Research Center for Ultra-High Voltage Electron Microscopy, Osaka University, 7-1 Mihogaoka, Ibaraki, \\ Osaka 567-0047, Japan
}

Biological materials such as cells and viruses are difficult to observe with sufficient contrast by transmission electron microscopy (TEM) because they have low interaction with the electron beam. Staining with heavy atoms can be used to increase the image contrast for these materials, but the process of staining degenerates the anatomy as a side effect. Therefore, an observation method for non-stained samples is required. Phase contrast TEM using a phase plate is considered a potential practical solution for this demand $[1,2,3]$. However, conventionally utilized phase plates-for example, electrostatic types or thin-film types - have difficulties for practical use due to static charging effect, damage to the phase plate itself, and deterioration of phase shift within an operation period.

To solve these problems, particularly the deterioration of phase shift, a contact-potential-type phase plate was proposed [4]. Figure 1 shows a schematic of the phase plate. A ring electrode has a three-metal layer (Metal 1/ Metal 2 / Metal 1), the outer Metal 1 layer is connected electrically to the ground, and the inner Metal 2 layer has a contact potential equal to the work function difference between Metal 1 and Metal 2. The contact potential generates an electric field inside the ring electrode and brings about a phase shift to the incident electron wave with an amount $\Delta \theta$ that can be expressed as

$$
\Delta \theta=\sigma \cdot\left(\phi_{2}-\phi_{1}\right) \cdot \mathrm{t}_{2}[\mathrm{rad}] \quad\left(\sigma=\frac{2 \pi}{\lambda} \cdot \frac{\left(\mathrm{m}_{0} \mathrm{c}^{2}+\mathrm{eV} \mathrm{ac}\right)}{\mathrm{V}_{\mathrm{ac}}\left(2 \mathrm{~m}_{0} \mathrm{c}^{2}+\mathrm{eV} \mathrm{ac}\right)}\left[\frac{\mathrm{rad}}{\mathrm{V} \cdot \mathrm{m}}\right]\right)
$$

where $\lambda$ is the wavelength of the electron, $c$ is the speed of light, $m_{0}$ is the electron mass, $e$ is the electron charge, $V_{a c}$ is an acceleration voltage, and $\sigma$ is an interaction constant between electron wave and electric field. As a value of work-function difference, $\left(\varphi_{2}-\varphi_{1}\right)$ is a constant value after two kinds of materials are chosen, and the phase shift can be controlled by the thickness of the Metal 2 layer of $t_{2}$. The contactpotential-type phase plate has three advantages: (1) a simple structure that enables a small size ring electrode, (2) the ability to generate an electric field without external power supply, and (3) an insulatorfree structure that suppresses electrostatic charging.

Figure 2(a) shows a scanning electron microscopy (SEM) image of our contact-potential-type phase plate fabricated using a focused ion beam (FIB) technique and vacuum deposition. The ring electrode was supported by three rods mechanically, and two kinds of metals, gold as Metal 1, and copper as Metal 2 were chosen for the materials. The phase shift of the electron wave, which is induced by the phase plate, can be directly measured by electron holography. Figure 2(b) shows a phase distribution around the ring electrode that clearly shows an electrostatic field formed only inside the ring electrode. Figure 2(c) shows the phase profile along the dotted line in Fig. 2(b); the phase difference is about $1.6 \cong \pi / 2$ rad. It was also confirmed that the phase shift was stable and did not deteriorate during several weeks of operation.

We installed the fabricated phase plate in a field emission TEM (HF-2000) as a substitute for an objective aperture. Figure 3 shows micrographs obtained from non-stained rat liver by the HF-2000 
operated at a $100-\mathrm{kV}$ acceleration voltage. An in-focus micrograph without the phase plate is shown in Fig. 3(a) and in-focus micrograph after the phase plate installation is shown in (b). Both micrographs were taken in the same area of the sample. The image contrast in (b) is obviously enhanced by using the phase plate, and the ribosome, which is aligned along the endoplasmic reticulum, becomes visible with high contrast. For our future work, we intend to extend the application to other kinds of practical materials in biology and organic chemistry.

[1] R. Danev et al.: Ultramicroscopy 88 (2001) 243.

[2] T. Matsumoto et al.: Ultramicroscopy 63 (1996) 5.

[3] R. Cambie et al.: Ultramicroscopy 107 (2007) 329.

[4] J. M. Perry-Houtsa1, et al.: Microsc. Microanal. 18 Suppl-S2 (2012) 476.

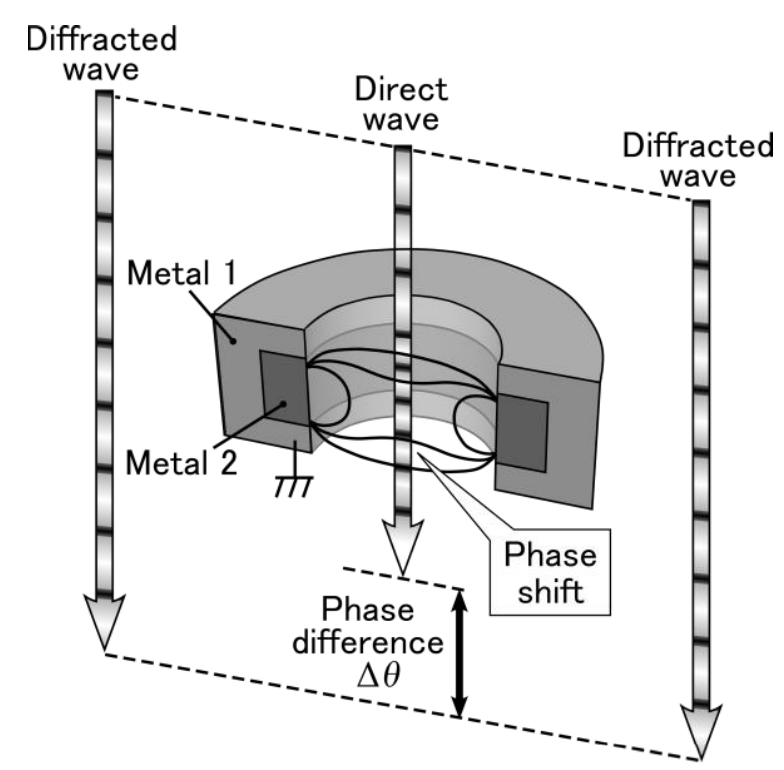

Figure 1. The cross-sectional structure of the phase plate.
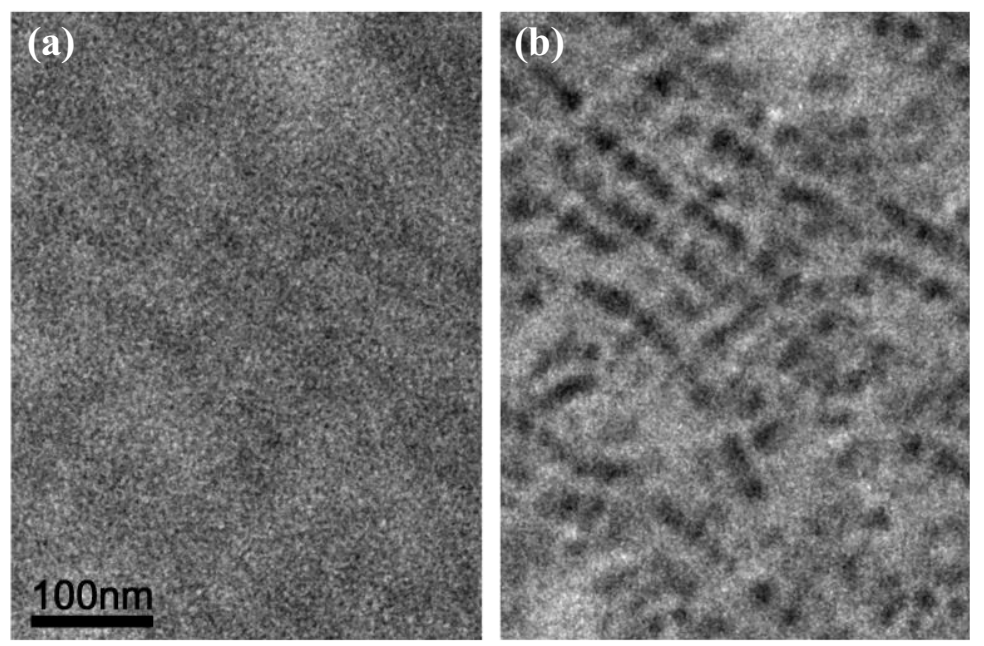

Figure 3. Electron micrographs from non-stained rat liver.

(a) Conventional micrograph without phase plate (in focus).

(b) Micrograph with phase plate (in focus). (a)

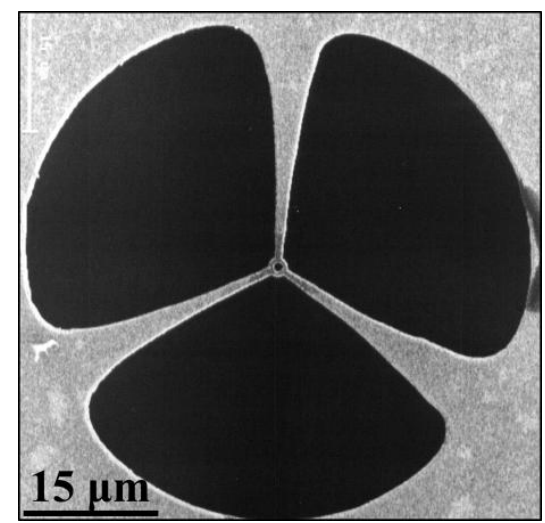

(b)

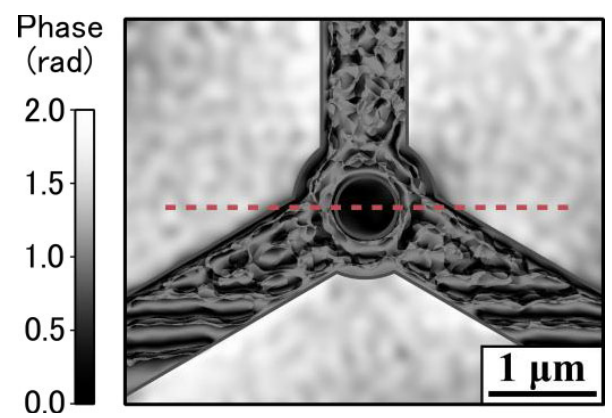

(c)

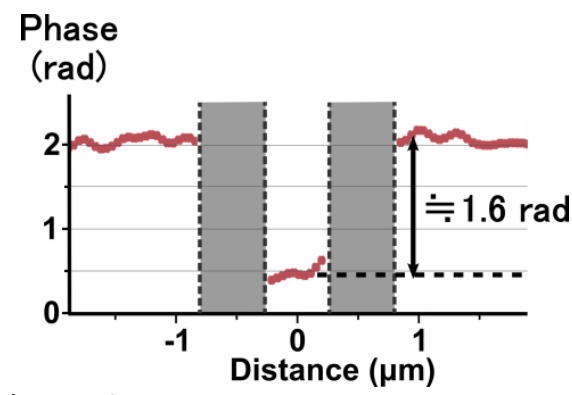

Figure 2.

(a) SEM image of the phase plate.

(b) Reconstructed phase distribution.

(c) Phase profile along dotted line in (b). 